Pacific Journal of Mathematics

TENSOR PRODUCTS OF BANACH BUNDLES

aston Kitchen, JR, and David A. Robbins 


\title{
TENSOR PRODUCTS OF BANACH BUNDLES
}

\author{
J. W. Kitchen AND D. A. RobBins
}

This paper is concerned with projective and inductive tensor products of bundles of Banach spaces. Let $\pi: E \rightarrow S$ and $\rho: F \rightarrow T$ be bundles of Banach spaces over the locally compact Hausdorff spaces $S$ and $T$, with fibers $\left\{E_{s}: s \in S\right\}$ and $\left\{F_{t}: t \in T\right\}$, respectively. Let $\Gamma_{0}(\pi)$ and $\Gamma_{0}(\rho)$ be their spaces of sections which disappear at infinity. We show the existence of a bundle $\pi \hat{\otimes} \rho: E \hat{\otimes} F \rightarrow S \times T$ whose fibers are $\left\{E_{s} \widehat{\otimes} E_{t}:(s, t) \in S \times T\right\} ;$ if $\sigma \in \Gamma_{0}(\pi)$ and $\tau \in \Gamma_{0}(\rho)$, then their pointwise tensor $\sigma \odot \tau$ defined by $(\sigma \odot \tau)(s, t)=\sigma(s) \otimes \tau(t)$ is a section of the bundle $\pi \hat{\otimes} \rho: E \hat{\otimes} F \rightarrow S \times T$. Further, we show the existence of a bundle $\pi \hat{\widehat{\otimes}} \rho: E \hat{\hat{\otimes}} F \rightarrow S \times T$ whose fibers are $\left\{E_{s} \hat{\hat{\otimes}} F_{t}:(s, t) \in S \times T\right\}$, and demonstrate that $\Gamma_{0}(\pi) \hat{\hat{\otimes}}$ $\Gamma_{0}(\rho)$ and $\Gamma_{0}(\pi \hat{\otimes} \rho)$ are isometrically isomorphic.

The present paper continues the study begun in [5] of the relationships between Banach modules and bundles of Banach spaces. Specifically, it concerns tensor products of such objects.

Given two bundles of Banach spaces $\pi: E \rightarrow S$ and $\rho: F \rightarrow T$ having locally compact base spaces we show that there is a bundle of Banach spaces $\theta: G \rightarrow S \times T$ having the following properties:

(1) for each pair $(s, t)$ in $S \times T$ the stalk $G_{s t}=\theta^{-1}(\{(s, t)\})$ is $E_{s} \hat{\otimes} F_{t}$, where, as in [5], $E_{s}=\pi^{-1}(\{s\})$ and $F_{t}=\rho^{-1}(\{t\})$;

(2) given two sections $\sigma \in \Gamma_{0}(\pi)$ and $\tau \in \Gamma_{0}(\rho)$ their pointwise tensor product $\sigma \odot \tau$ defined by

$$
(\sigma \odot \tau)(s, t)=\sigma(s) \otimes \tau(t)
$$

is a section of the bundle $\theta: G \rightarrow S \times T$. (Here again the reader is referred to [5] for notation and terminology.)

The bundle $\theta: G \rightarrow S \times T$ is called the projective tensor product of the given bundles and is denoted by $\pi \hat{\otimes} \rho: E \hat{\otimes} F \rightarrow S \times T$.

Tensor products of Banach bundles relate to tensor products of Banach modules in the following fashion. Suppose that $A$ and $B$ are commutative Banach algebras with maximal ideals spaces $S$ and $T$. Suppose further that $(M, A)$ and $(N, B)$ are Banach modules which satisfy the (KR) condition. Then

(1) the Banach module $(M \hat{\otimes} N, A \hat{\otimes} B)$ also satisfies the (KR) condition;

(2) the canonical bundle associated with $(M \hat{\otimes} N, A \hat{\otimes} B)$ is (bundle isomorphic to) the tensor product of the canonical bundles 
of the given modules;

(3) for any elements $x \in M$ and $y \in N$ the Gelfand transform of $x \otimes y$ is the pointwise tensor product of the transforms $\hat{x}$ and $\hat{y}$.

The order in which these results are developed is possibly not what one might expect. In the first section a study is begun of the canonical bundle and Gelfand representation of the tensor product module $(M \hat{\otimes} N, A \hat{\otimes} B)$. In the the second section, the tensor product bundle is defined essentially to be the canonical bundle of $\left(\Gamma_{0}(\pi) \hat{\otimes} \Gamma_{0}(\rho), C_{0}(S) \hat{\otimes} C_{0}(T)\right)$. Afterwards, the results of section one are reinterpreted in terms of tensor products of bundles. Section 3 treats further properties of projective tensor products, while the fourth and final section deals with inductive tensor products. Given two bundles of Banach spaces $\pi: E \rightarrow S$ and $\rho: F \rightarrow T$ with locally compact base spaces, their inductive tensor product $\pi \hat{\hat{\otimes}} \rho: E \hat{\hat{\otimes}} F \rightarrow$ $S \times T$ is constructed and it is shown that there is a natural isomor$\operatorname{phism} \Gamma_{0}(\pi) \hat{\hat{\otimes}} \Gamma_{0}(\rho) \cong \Gamma_{0}(\pi \hat{\hat{\otimes}} \rho)$.

1. Tensor products of Banach modules and their Gelfand representations. In this section we shall begin to study the Gelfand representation of a tensor product of Banach modules. We prove first that the $(\mathrm{KR})$ condition is preserved under the formation of tensor products.

Suppose $(M, A)$ is a Banach module, where $A$ is a commutative Banach algebra with maximal ideal space $S$. If $s \in S$, we denote by $I_{\mathrm{s}}$ the maximal ideal associated with $s$ and by $J_{s}$ the closed linear span of the set $\left\{a x: a \in I_{s}, x \in M\right\}$. As in [5], we also denote by $\left(M^{*}\right)_{s}$ the set of all functionals $F$ in $M^{*}$ for which the identity

$$
F(a x)=\widehat{a}(s) F(x)
$$

holds for all $a \in A$ and $x \in M$. The module $(M, A)$ is said to satisfy the (KR) condition if for each $s \in S,\left(J_{s}\right)^{\perp}$, the annihilator of $J_{s}$ in $M^{*}$, is equal to $\left(M^{*}\right)_{s}$. Since the inclusion $\left(M^{*}\right)_{s} \subset\left(J_{s}\right)^{\perp}$ always holds, the $(\mathrm{KR})$ condition is equivalent to the inclusions $\left(J_{s}\right)^{\perp} \subset\left(M^{*}\right)_{s}$ for all $s \in S$.

Proposition 1.1. Let $A$ and $B$ be commutative Banach algebras with maximal idal spaces $S$ and $T$ respectively. If $(M, A)$ and $(N, B)$ are Banach modules which satisfy the (KR) condition, then the tensor product module $(M \hat{\otimes} N, A \hat{\otimes} B)$ also satisfies the (KR) condition.

Proof. Recall that the maximal ideal space of $A \hat{\otimes} B$ can be identified with $S \times T$ in such a way that 


$$
(a \otimes b)^{\wedge}(s, t)=\hat{a}(s) \hat{b}(t)
$$

for all $a \in A, b \in B, s \in S$, and $t \in T$. Recall also that the way in which $M \otimes N$ is a module over $A \otimes B$ is characterized by the identity:

$$
(a \otimes b)(x \otimes y)=(a x) \otimes(b y),
$$

which holds for all $a \in A, b \in B, x \in M$, and $y \in N$.

Let $(s, t) \in S \times T$ be arbitrary. We denote by $I_{s t}$ the maximal ideal in $A \hat{\otimes} B$ corresponding to $(s, t)$ and by $J_{s t}$ the closed linear span of $\left\{c z: c \in I_{s t}, z \in M \hat{\otimes} N\right\}$. We must show that $\left(J_{s t}\right)^{\perp} \subset\left((M \hat{\otimes} N)^{*}\right)_{s t}$. Suppose, then, that $F \in\left(J_{s t}\right)^{\perp}$. Because of the linearity and continuity of $F$ it suffices to show that

$$
\begin{aligned}
F((a \otimes b)(x \otimes y)) & =(a \otimes b)^{\wedge}(s, t) F(x \otimes y) \\
& =\widehat{a}(s) \hat{b}(t) F(x \otimes y)
\end{aligned}
$$

for all $a \in A, b \in B, x \in M$, and $y \in N$.

We let $R_{F}$ be the element of $\operatorname{Hom}\left(M, N^{*}\right)$ associated with $F$, that is,

$$
F(x \otimes y)=\left\langle y, R_{F}(x)\right\rangle,
$$

for all $x \in M$ and $y \in N$. Similarly, we let $R_{F}^{\prime}$ be the element of $\operatorname{Hom}\left(N, M^{*}\right)$ associated with $F$, that is, $F(x \otimes y)=\left\langle x, R_{F}^{\prime}(y)\right\rangle$. If $b \in I_{t}$ and $a \in A$, then $(a \otimes b)^{\wedge}(s, t)=\widehat{a}(s) \hat{b}(t)=0$, so $(a \otimes b) \in I_{s t}$ and thus

$$
\left\langle b y, R_{F}(a x)\right\rangle=F\left(a x^{\prime} \otimes b y\right)=F((a \otimes b)(x \otimes y))=0
$$

for all $(x, y) \in M \times N$. Thus, $R_{F}(a x) \in\left(J_{t}\right)^{\perp}=\left(N^{*}\right)_{t}$ for all $a \in A$ and $x \in M$. Hence for arbitrary $a \in A, b \in B, x \in M, y \in N$ we have

$$
\begin{aligned}
F(a x \otimes b y) & =\left\langle b y, R_{F}(a x)\right\rangle=\hat{b}(t)\left\langle y, R_{F}(a x)\right\rangle \\
& =\hat{b}(t) F(a x \otimes y) .
\end{aligned}
$$

Since $(M, A)$ satisfies the (KR) condition, the module is essential, and it then follows from $\left(^{*}\right)$ that

$$
F(x \otimes b y)=\hat{b}(t) F(x \otimes y)
$$

for all $x \in M, b \in B$, and $y \in N$. Similarly, using $R_{F}^{\prime}$, one can show that

$$
F(a x \otimes y)=\hat{a}(s) F(x \otimes y)
$$

for all $a \in A, x \in M$, and $y \in N$. Finally, we have

$$
\begin{aligned}
F((a \otimes b)(x \otimes y)) & =F(a x \otimes b y)=\hat{b}(t) F(a x \otimes y) \\
& =\hat{a}(s) \hat{b}(t) F(x \otimes y),
\end{aligned}
$$


for all $a \in A, b \in B, x \in M, y \in N$.

Thus, if the modules $(M, A)$ and $(N, B)$ satisfy the hypotheses of Proposition 1.1, then we can consider the canonical bundles and Gelfand representations for the modules $(M, A),(N, B)$, and $(M \hat{\otimes} N$, $A \hat{\otimes} B)$. Let us denote by $\pi: E \rightarrow S, \rho: F \rightarrow T$, and $\theta: H \rightarrow S \times T$ respectively the canonical bundles for these three modules. We will show that the stalk $H_{s t}=\theta^{-1}(\{(s, t)\})$ is isomorphic to the tensor product of the stalks $E_{s}=\pi^{-1}(\{s\})$ and $F_{t}=\rho^{-1}(\{t\})$. In doing so, we make use of a lemma concerning bilinear maps on quotient spaces.

Lemma 1.2. Suppose we are given the followiug:

(1) Banach spaces $X, Y$, and $Z$;

(2) a bounded bilinear map $f: X \times Y \rightarrow Z$;

(3) subsets $M \subset X$ and $N \subset Y$ such that

$$
f(x, y)=0 \text { for all } x \in M \text { and } y \in Y
$$

and

$$
f(x, y)=0 \text { for all } x \in X \text { and } y \in N .
$$

Then, if we denote by $M^{\prime}$ and $N^{\prime}$ the closed linear spans of $M$ and $N$ respectively, it follows that there exists a unique bounded bilinear map

$$
\tilde{f}:\left(X / M^{\prime}\right) \times\left(Y / N^{\prime}\right) \longrightarrow Z
$$

such that

$$
\widetilde{f}\left(x+M^{\prime}, y+N^{\prime}\right)=f(x, y),
$$

for all $x \in X$ and $y \in Y$. Moreover, $\widetilde{f}$ has the same norm as $f$.

The proof is straightforward and is therefore omitted.

Proposition 1.3. Suppose that the hypotheses of Proposition 1.1 are satisfied. Let $\pi: E \rightarrow S, \rho: F \rightarrow T$, and $\theta: H \rightarrow S \times T$ be the canonical bundles corresponding to the modules $(M, A),(N, B)$, and $(M \hat{\otimes} N, A \hat{\otimes} B)$ respectively. For each pair $(s, t)$ in $S \times T$ therc exists an isometric isomorphism $\phi_{s t}: E_{s} \hat{\otimes} F_{t} \rightarrow H_{s t}$ such that

$$
\dot{\phi}_{s t}(\widehat{x}(s) \otimes \widehat{y}(t))=(x \otimes y)^{\wedge}(s, t)
$$

for all $x \in M$ and $y \in N$.

Proof. We define a map $f: M \times N \rightarrow H_{s t}$ by setting 


$$
f(x, y)=(x \otimes y)^{\wedge}(s, t) .
$$

Then $f$ is clearly a bilinear map with norm one or less. Moreover, for all $a \in A, b \in B, x \in M$, and $y \in N$ we have

$$
\begin{aligned}
f(a x, b y) & =(a x \otimes b y)^{\wedge}(s, t) \\
& =((a \otimes b)(x \otimes y))^{\wedge}(s, t) \\
& =(a \otimes b)^{\wedge}(s, t)(x \otimes y)^{\wedge}(s, t) \\
& =\hat{a}(s) \hat{b}(t)(x \otimes y)^{\wedge}(s, t) .
\end{aligned}
$$

It is clear, then, that $f(a x, b y)=0$ if either $a \in I_{s}$ or $b \in I_{t}$. Because $(M, A)$ and $(N, B)$ are essential modules, it follows that

$$
f(a x, y)=0 \text { for all } a \in I_{s}, x \in M, y \in N
$$

and

$$
f(x, b y)=0 \text { for all } b \in I_{t}, x \in M, y \in N .
$$

By the previous lemma, it follows that there is a unique bilinear map

$$
\tilde{f}: E_{s} \times F_{t}=\left(M / J_{s}\right) \times\left(N / J_{t}\right) \longrightarrow H_{s t}
$$

such that $\|\tilde{f}\|=\|f\| \leqq 1$ and

$$
\widetilde{f}(\widehat{x}(s), \widehat{y}(t))=\widetilde{f}\left(x+J_{s}, y+J_{t}\right)=f(x, y)=(x \otimes y)^{\wedge}(s, t),
$$

for all $x \in M, y \in N$. By the universal mapping property of tensor products it follows, finally, that there is a linear map $\phi: E_{s} \hat{\otimes} F_{t} \rightarrow$ $H_{s t}$ such that $\|\boldsymbol{\phi}\|=\|\widetilde{f}\| \leqq 1$ and

$$
\phi(\hat{x}(s) \otimes \hat{y}(t))=\widetilde{f}(\hat{x}(s), \hat{y}(t))=(x \otimes y)^{\wedge}(s, t)
$$

for all $x \in M, y \in N$.

We next define a map $\psi: H_{s t} \rightarrow E_{s} \hat{\otimes} F_{t}$ which will turn out to be the inverse of $\phi$. We do this in stages. First we define a map $g: M \times N \rightarrow E_{s} \hat{\otimes} F_{t}$ by setting

$$
g(x, y)=\hat{x}(s) \otimes \hat{y}(t) .
$$

Clearly, $g$ is bilinear and $\|g\| \leqq 1$. It follows that there is a unique linear map $\widetilde{g}: M \hat{\otimes} N \rightarrow E_{s} \hat{\otimes} F_{t}$ such that $\|\widetilde{g}\|=\|g\| \leqq 1$ and

$$
\widetilde{g}(x \otimes y)=g(x, y)=\widehat{x}(s) \otimes \widehat{y}(t) .
$$

In addition, the function $\widetilde{g}$ has the following property:

$$
\widetilde{g}(c z)=\hat{c}(s, t) \widetilde{g}(z)
$$

for all $c \in A \hat{\otimes} B$ and all $z \in M \hat{\otimes} N$. Because of the linearity and continuity of $\widetilde{g}$, it suffices to check $\left(^{*}\right)$ when $c$ is of the form $a \otimes b$ 
and $z$ is of the form $x \otimes y$, and that is easily done:

$$
\begin{aligned}
\widetilde{g}((a \otimes b)(x \otimes y)) & =\widetilde{g}(a x \otimes b y) \\
& =\widehat{a x}(s) \otimes \widehat{b y}(t)=\widehat{a}(s) \hat{b}(t) \hat{x}(s) \otimes \widehat{y}(t) \\
& =(a \otimes b)^{\wedge}(s, t) \widetilde{g}(x \otimes y) .
\end{aligned}
$$

From $\left({ }^{*}\right)$, it follows that $\widetilde{g}(c z)=0$ whenever $\widehat{c}(s, t)=0$, that is, $c \in$ $I_{s t}$. Thus, $\widetilde{g}(z)=0$ whenever $z$ belongs to $J_{s t}$, the closed linear span of $\left\{c z: c \in I_{s t}, z \in M \otimes N\right\}$. Hence there is a unique linear map

$$
\psi: H_{s t}=(M \hat{\otimes} N) / J_{s t} \longrightarrow E_{s} \hat{\otimes} F_{t}
$$

such that $\|\psi\|=\|\widetilde{g}\| \leqq 1$ and

$$
\psi\left((x \otimes y)^{\wedge}(s, t)\right)=\psi\left(x \otimes y+J_{s t}\right)=\widetilde{g}(x \otimes y)=\widehat{x}(s) \otimes \widehat{y}(t),
$$

for all $x \in M$ and $y \in N$.

The rest is easy. One checks that $\psi \circ \phi$ and $\phi \circ \psi$ are the identity on $E_{s} \hat{\otimes} F_{t}$ and $H_{s t}$ respectively. Thus, the map $\phi: E_{s} \hat{\otimes} F_{t} \rightarrow H_{s t}$ is bijective and $\psi$ is its inverse. Furthermore, since $\phi$ and $\psi$ are both norm decreasing, they are, in fact norm preserving.

2. The construction of projective tensor products of bundles. We now apply Proposition 1.3 to construct the tensor product of Banach bundles.

THEOREM 2.1. Let $\pi: E \rightarrow S$ and $\rho: F \rightarrow T$ be bundles of Banach spaces for which the base spaces $S$ and $T$ are locally compact Hausdorff. Then there exists a unique bundle of Banach spaces $\phi: G \rightarrow$ $S \times T$ with the following properties:

(1) for each pair $(s, t)$ in $S \times T$ the stalk $G_{s t}=\theta^{-1}(\{(s, t)\})$ is $E_{s} \hat{\otimes} F_{t}$, where $E_{s}=\pi^{-1}(\{s\})$ and $F_{t}=\rho^{-1}(\{t\})$;

(2) $\theta: G \rightarrow S \times T$ is bundle isomorphic to the canonical bundle for the module $\left(\Gamma_{0}(\pi) \hat{\otimes} \Gamma_{0}(\rho), C_{0}(S) \hat{\otimes} C_{0}(T)\right)$; more precisely, if $\theta^{\prime}: H \rightarrow S \times T$ is the canonical bundle for the latter module then there exists a norm preserving bundle isomorphism $\phi: G \rightarrow H$ such that

$$
\phi(\sigma(s) \otimes \tau(t))=(\sigma \otimes \tau)^{\wedge}(s, t)
$$

for all $\sigma \in \Gamma_{0}(\pi), \tau \in \Gamma_{0}(\rho), s \in S, t \in T$.

(3) the tensor map $\otimes: E \times F \rightarrow G$, which assigns to each pair $(x, y)$ in $E \times F$ its tensor $x \otimes y$ in the stalk $E_{\pi(x)} \hat{\otimes} F_{\rho(y)}$, is continuous.

Proof. We can identify the given bundle $\pi: E \rightarrow S$ with the 
canonical bundle the module $\left(\Gamma_{0}(\pi), C_{0}(S)\right)$ in such a way that the Gelfand representation of the module is simply the identity map. (See [5], §3.) The same is true, of course, for the bundle $\rho: F \rightarrow T$. If we denote by $\theta^{\prime}: H \rightarrow S \times T$ the canonical bundle for the module

$$
\left(\Gamma_{0}(\pi) \hat{\otimes} \Gamma_{0}(\rho), C_{0}(S) \hat{\otimes} C_{0}(T)\right),
$$

then for each pair $(s, t)$ in $S \times T$ Proposition 1.3 assures us of the existence of a unique isometric isomorphism $\phi_{s t}: E_{s} \hat{\otimes} F_{t} \rightarrow H_{s t}$ such that

$$
\phi_{s t}(\sigma(s) \otimes \tau(t))=\dot{\phi}_{s t}(\hat{\sigma}(s) \otimes \hat{\tau}(t))=(\sigma \otimes \tau)^{\wedge}(s, t)
$$

for all $\sigma \in \Gamma_{0}(\pi)$ and $\tau \in \Gamma_{0}(\rho)$. If we now let $G$ be the disjoint union of the family of Banach spaces $\left\{E_{s} \hat{\otimes} F_{t}: s \in S, t \in T\right\}$, then we have a bijection $\phi: G \rightarrow H$ whose restrictions to stalks are the maps $\dot{\phi}_{s t}$. We topologize $G$ by transplanting the topology from $H$ to $G$ via the map $\dot{\phi}^{-1}$. Then $\theta: G \rightarrow S \times T$ becomes a bundle of Banach spaces $(\theta$ being the natural surjection), $\phi$ becomes an isometric bundle isomorphism, and (2) is obviously satisfied.

Thus, if we identify $G$ and $H$ via $\phi$, then the Gelfand transform of $\sigma \otimes \tau$ is simply the pointwise tensor product $\sigma \odot \tau$ as defined in the introduction, that is,

$$
(\sigma \otimes \tau)^{\wedge}(s, t)=\sigma(s) \otimes \tau(t)=(\sigma \otimes \tau)(s, t) .
$$

In proving continuity of the tensor map $\otimes: E \times F \rightarrow G$ we shall make this identification.

Let $(x, y)$ be an arbitrary element of $E \times F$. We will show that $\otimes$ is continuous at $(x, y)$. Set $s=\pi(x)$ and $t=\rho(y)$ and choose sections $\sigma \in \Gamma_{0}(\pi)$ and $\tau \in \Gamma_{0}(\rho)$ such that $\sigma(s)=x, \tau(t)=y,\|\sigma\|=$ $\|x\|$, and $\|\tau\|=\|y\|$. (See [5], Corollary 1.2.) Then the section $(\sigma \otimes \tau)^{\wedge}=\sigma \odot \tau$ passes through $x \otimes y$, that is,

$$
(\sigma \odot \tau)(s, t)=\sigma(s) \otimes \tau(t)=x \otimes y .
$$

Consider now a neighborhood of $x \otimes y$. We may assume that it is of the form

$$
\mathscr{W}=\{z \in G:\|z-(\sigma \odot \tau)(\theta(z))\|<\varepsilon, \theta(z) \in W\},
$$

where $W$ is a neighborhood of $(s, t)$. Inside $W$ is a neighborhood of the form $U \times V$, where $U$ is a neighborhood of $s$ and $V$ is a neighborhood of $t$. We then set

$$
\mathscr{\mathscr { C }}=\left\{x^{\prime} \in E:\left\|x^{\prime}-\sigma\left(\pi\left(x^{\prime}\right)\right)\right\|<\varepsilon_{1}, \pi\left(x^{\prime}\right) \in U\right\}
$$




$$
\mathscr{C}=\left\{y^{\prime} \in F:\left\|y^{\prime}-\tau\left(\rho\left(y^{\prime}\right)\right)\right\|<\varepsilon_{2}, \rho\left(y^{\prime}\right) \in V\right\},
$$

where $\varepsilon_{1}=\varepsilon / 2(\|y\|+1)$ and $\varepsilon_{2}=1 / 2 \min (1, \varepsilon /(\|x\|+1))$. Then $\mathscr{U} \times \mathscr{V}$ is a neighborhood of $(x, y)$. Suppose, then, that $\left(x^{\prime}, y^{\prime}\right) \in \mathscr{U} \times \mathscr{Y}$. If we set $s^{\prime}=\pi\left(x^{\prime}\right)$ and $t^{\prime}=\rho\left(y^{\prime}\right)$, then $s^{\prime} \in U$ and $t^{\prime} \in V$, so that $\theta\left(x^{\prime} \otimes y^{\prime}\right)=\left(s^{\prime}, t^{\prime}\right)$ belongs to $W$. Furthermore,

$$
\begin{aligned}
\left\|y^{\prime}\right\| & \leqq\left\|y^{\prime}-\tau\left(\rho\left(y^{\prime}\right)\right)\right\|+\left\|\tau\left(\rho\left(y^{\prime}\right)\right)\right\| \\
& \leqq \varepsilon_{2}+\|\tau\| \leqq 1+\|y\|
\end{aligned}
$$

and

$$
\begin{aligned}
\| x^{\prime} \otimes & y^{\prime}-(\sigma \odot \tau)\left(\theta\left(x^{\prime} \otimes y^{\prime}\right)\right) \| \\
= & \left\|x^{\prime} \otimes y^{\prime}-\sigma\left(s^{\prime}\right) \otimes \tau\left(t^{\prime}\right)\right\| \\
\leqq & \left\|x^{\prime} \otimes y^{\prime}-\sigma\left(s^{\prime}\right) \otimes y^{\prime}\right\| \\
& +\left\|\sigma\left(s^{\prime}\right) \otimes y^{\prime}-\sigma\left(s^{\prime}\right) \otimes \tau\left(t^{\prime}\right)\right\| \\
= & \left\|x^{\prime}-\sigma\left(\pi\left(x^{\prime}\right)\right)\right\| \quad\left\|y^{\prime}\right\| \\
& +\left\|\sigma\left(s^{\prime}\right)\right\|\left\|y^{\prime}-\tau\left(\rho\left(y^{\prime}\right)\right)\right\| \\
< & \varepsilon_{1}(1+\|y\|)+\|x\| \varepsilon_{2}<\varepsilon .
\end{aligned}
$$

Hence $x^{\prime} \otimes y^{\prime}$ belongs to the neighborhood $\mathscr{Y}$ of $x \otimes y$. This proves continuity of the tensor map at $(x, y)$.

The bundle $\theta: G \rightarrow S \times T$ will be called the projective tensor product of the given bundles $\pi: E \rightarrow S$ and $\rho: F \rightarrow T$, and we will henceforth denote it by $\pi \hat{\otimes} \rho: E \hat{\otimes} F \rightarrow S \times T$. Note that if we have two sections $\sigma \in \Gamma_{0}(\pi)$ and $\tau \in \Gamma_{0}(\rho)$, then, as we observed in the previous proof, their pointwise tensor product $\sigma \odot \tau$, defined by

$$
(\sigma \odot \tau)(s, t)=\sigma(s) \otimes \tau(t),
$$

is a section of the tensor product bundle. Moreover, because of the continuity of the tensor map $\otimes: E \times F \rightarrow E \hat{\otimes} F$, it follows that the pointwise tensor product of two local sections (possibly unbounded) is a local section of the tensor product bundle.

We can now reinterpret Proposition 1.3.

THEOREM 2.2. Let $A$ and $B$ be commutative Banach algebras with maximal ideal spaces $S$ and $T$ respectively. Suppose that $(M, A)$ and $(N, B)$ are normed modules which satisfy the (KR) condition. Then the module $(M \hat{\otimes} N, A \hat{\otimes} B)$ also satisfies the (KR) condition and the canonical bundle for this product module can be naturally identified with the projective tensor product of the canonical bundles of the given modules. So identified, the Gelfand transform of an 
element in $M \hat{\otimes} N$ of the form $x \otimes y$ is simply the pointwise tensor product of the transformations $\hat{x}$ and $\hat{y}$.

Note that the Gelfand representation ${ }^{\wedge}: M \hat{\otimes} N \rightarrow \Gamma_{0}(\pi \hat{\otimes} \rho)$ need not be surjective. For example, let $S$ and $T$ be infinite compact Hausdorff spaces. Then $C(S)$ and $C(T)$ are (may be identified with) spaces of sections of bundles $\pi: E \rightarrow S$ and $\rho: F \rightarrow T$, all of whose stalks are $C$. With this identification, the sectional Gelfand representation of an element in $C(S) \hat{\otimes} C(T)$ of the form $f \otimes g$ is simply the function $f g \in C(S \times T)$, where $(f g)(s, t)=f(s) g(t)$. Thus, the range of the Gelfand representation is the proper subspace of $C(S \times T)$ consisting of all functions expressible as the sum of series of products $f g$.

Nor need the Gelfand representation ${ }^{\wedge}: M \hat{\otimes} N \rightarrow \Gamma_{0}(\pi \hat{\otimes} \rho)$ be injective, even when both ${ }^{\wedge}: M \rightarrow \Gamma_{0}(\pi)$ and ${ }^{\wedge}: N \rightarrow \Gamma_{0}(\rho)$ are injective. To see this, let $A$ and $B$ be commutative semisimple Banach algebras with identities such that $A \hat{\otimes} B$ is not semisimple. (See [6].) The sectional Gelfand representation of the module $(A \hat{\otimes} B, A \hat{\otimes} B)$ is (may be identified with) the classical Gelfand representation of $A \hat{\otimes} B$, and so the sectional Gelfand representation is not injective.

3. Further results. The first theorem in this section of the paper is analogous to results in Gelbaum [3]. Since the proofs are also similar, they are omitted.

THeOREM 3.1. Suppose that $(M, A)$ and $(N, B)$ are modules which satisfy the hypotheses of Theorem 2.2.

(1) For all $x \in M$ and $y \in N$ the support of $(x \otimes y)^{\wedge}$ is the Cartesian product of the supports of $\hat{x}$ and $\hat{y}$.

(2) If the sets $\{x \in M: \operatorname{supp} \hat{x}$ is compact $\}$ and $\{y \in N: \operatorname{supp} \hat{y}$ is compact $\}$ are dense in $M$ and $N$ respectively, then the set $\{z \in M \hat{\otimes}$ $N$ : supp $\hat{z}$ is compact\} is dense in $M \hat{\otimes} N$.

If $M$ and $N$ are Banach modules over a commutative Banach algebra $A$, then one can form their $A$-tensor product, $M \otimes_{A} N$, which is defined to be the quotient space $(M \hat{\otimes} N) / K$, where $K$ is the smallest closed subspace containing all elements of the form $(a x) \otimes y-x \otimes$ $(a y)$ (where $a \in A, x \in M$, and $y \in N$ ). Given $x \in M$ and $y \in N, x \bigotimes_{A} y$ is defined to be the coset $x \otimes y+K$. Clearly, for all $a \in A, x \in M$, and $y \in N$

$$
(a x) \bigotimes_{A} y=x \boldsymbol{\bigotimes}_{A}(a y)
$$

moreover, $M \otimes_{A} N$ can be made into a Banach $A$-module in just one 
way so that both sides of the preceding equation are equal to $a\left(x \otimes_{A} y\right)$. (See Rieffel [7].) For such modules we have the following analogue of Theorem 2.2.

THEOREM 3.2. Let $A$ be a commutative Banach algebra with maximal ideal space $S$. Suppose that $(M, A)$ and $(N, A)$ are Banach modules which satisfy the (KR) condition and denote by $\pi: E \rightarrow S$ and $\rho: F \rightarrow S$ their respective canonical bundles. Then the module $\left(M \otimes_{A} N, A\right)$ also satisfies the $(\mathrm{KR})$ condition and its canonical bundle can be identified with the product bundle $\pi \hat{\otimes} \rho: E \hat{\otimes} F \rightarrow$ $S \times S$ restricted to the diagonal of $S \times S$. More precisely, if $\theta: H \rightarrow$ $S$ denotes the canonical bundle of $\left(M \otimes_{A} N, A\right)$, then for each $s \in S$ there is an isometric isomorphism $\phi_{s}: H_{s} \rightarrow E_{s} \hat{\otimes} F_{s}$ such that

$$
\phi_{s}\left(\left(x \otimes_{A} y\right)^{\wedge}(s)\right)=\widehat{x}(s) \otimes \widehat{y}(s)
$$

for all $x \in M$ and $y \in N$.

The next series of results concerns tensor products of bundles and their sections. We begin with a variant of Tietze's Extension Theorem.

Lemma 3.3 (Tietze's Extension Theorem for Sections). Let $\pi: E \rightarrow S$ be a bundle of Banach spaces, where $S$ is a compact Hausdorff space. Let $K$ be a closed subset of $S$. Then every local section $\sigma: K \rightarrow E$ can be extended without increase of norm to a global section $\tilde{\sigma}: S \rightarrow E$.

Proof. Let $M$ be the set consisting of restrictions to $K$ of global sections of the bundle $\pi: E \rightarrow S$. If $\sigma \in M$, and if $f \in C(K)$, then $\sigma$ is the restriction of some global section $\widetilde{\sigma}$, while $f$ is the restriction of some $\widetilde{f} \in C(S)$ (by the usual Tietze Extension Theorem). It follows that $f \sigma$ is the restriction to $K$ of the global section $\tilde{f} \widetilde{\sigma}$, so that $f \sigma \in M$. Thus, $M$ is a $C(K)$-submodule of $\Gamma(\pi \uparrow K)$, where $\Gamma(\pi \uparrow K)$ denotes the sections of the restricted bundle $\pi \uparrow K: E \cap \pi^{-1}(K) \rightarrow K$. Moreover, since $\pi: E \rightarrow S$ is a full bundle, so is the restricted bundle, so that $M$ is dense in $\Gamma(\pi \uparrow K)$ by the Stone-Weierstrass theorem for sections. (See, for example, [4].)

To show that $M$ is actually all of $\Gamma(\pi \uparrow K)$ and to complete the proof it suffices to prove the following: if $\sigma \in M$, then there exists a $\tilde{\sigma} \in \Gamma(\pi)$ such that $\sigma=\tilde{\sigma} \uparrow K$ and $\|\tilde{\sigma}\|=\|\sigma\|$. (That $M$ is closed and hence all of $\Gamma(\pi \uparrow K)$ will then follow from the completeness of the spaces $\Gamma(\pi \uparrow K)$ and $\Gamma(\pi)$.) To extend $\sigma \in M$ appropriately we proceed as follows: We first select any section $\widetilde{\sigma} \in \Gamma(\pi)$ (with possibly 
larger norm than $\sigma$ ) with $\tilde{\sigma}\lceil K=\sigma$. For each positive integer $n$ we set

$$
V_{n}=\left\{s \in S:\|\widetilde{\sigma}(s)\|<\frac{2^{n}}{2^{n}-1}\|\sigma\|\right\}
$$

Then $V_{n}$ is an open neighborhood of $K$, and we may choose a continuous function $f_{n}: S \rightarrow[0,1]$ such that $f_{n}$ has the value 1 on $K$ and the value zero off $V_{n}$. We set

$$
f=\sum_{n=1}^{\infty} 2^{-n} f_{n} \text { and } \tilde{\tau}=f \widetilde{\sigma} .
$$

It may then be easily verified that $\tilde{\tau}$ is a global section extending $\sigma$ and that $\|\tilde{\tau}(s)\|<\|\sigma\|$ whenever $s$ lies outside $K$. Thus, $\|\tilde{\tau}\|=$ $\|\sigma\|$.

(Compare this lemma with a weaker version in Fell [2].)

Theorem 3.4. Suppose that $\pi: E \rightarrow S$ and $\rho: F \rightarrow T$ are bundles of Banach spaces, where $S$ and $T$ are compact Hausdorff. Then each section of the product bundle $\pi \hat{\otimes} \rho: E \hat{\otimes} F \rightarrow S \times T$ can be uniformly approximated by sums of sections of the form $\sigma \odot t$, where $\sigma \in \Gamma(\pi)$ and $\tau \in \Gamma(\rho)$.

Proof. Let $M$ be the closure in $\Gamma(\pi \hat{\otimes} \rho)$ of finite sums of sections of the form $\sigma \odot \tau$. Clearly, $M$ is a closed subspace of $\Gamma(\pi \hat{\otimes} \rho)$. It is, moreover, a $C(S \times T)$-submodule: for if $\omega \in M$ and $h \in C(S \times T)$, then $\omega$ can be approximated by sections of the form $\sum_{k} \sigma_{k} \odot \tau_{k}$ and $h$ can be approximated by functions of the form $\sum_{j} f_{j} \odot g_{j}$, where $f_{j} \in C(S)$ and $g_{j} \in C(T)$ for each $j$, and where $\left(f_{j} \odot g_{j}\right)(s, t)=f_{j}(s) g_{j}(t)$. Hence $h \omega$ can be approximated by sections of the form

$$
\left(\sum_{j} f_{j} \odot g_{j}\right)\left(\sum_{k} \sigma_{k} \odot \tau_{k}\right)=\sum_{j, k}\left(f_{j} \sigma_{k}\right) \odot\left(g_{j} \tau_{k}\right),
$$

so that $h \omega \in M$. The conclusion now follows again by the bundle version of the Stone-Weierstrass theorem.

Corollary 3.5. Suppose that $\pi: E \rightarrow S$ and $\rho: F \rightarrow T$ are bundles of Banach spaces, where $S$ and $T$ are locally compact Hausdorff spaces. Then every section in $\Gamma_{0}(\pi \hat{\otimes} \rho)$ can be uniformly approximated by sums of sections of the form $\sigma \odot \tau$, where $\sigma \in \Gamma_{0}(\pi)$ and $\tau \in \Gamma_{0}(\rho)$. 
Proof. It suffices to prove the theorem for compactly supported sections in $\Gamma_{0}(\pi \hat{\otimes} \rho)$, since they are dense in $\Gamma_{0}(\pi \hat{\otimes} \rho)$.

Consider, then, a section $\omega \in \Gamma_{0}(\pi \hat{\otimes} \rho)$ which is supported on a compact set $K$. We may assume that $K=S^{\prime} \times T^{\prime}$, where $S^{\prime}$ and $T^{\prime \prime}$ are compact. (We can take $S^{\prime}$ and $T^{\prime}$ to be the coordinate projections of the original set $K$ onto $S$ and $T$ respectively.) Let $\varepsilon>0$ be arbitrary. By the theorem we can find sections $\sigma_{i}: S^{\prime} \rightarrow E$ and $\tau_{i}: T^{\prime} \rightarrow F$ such that

$$
\left\|\omega-\sum \sigma_{i} \odot \tau_{i}\right\|<\varepsilon / 2
$$

on the set $S^{\prime} \times T^{\prime}$. By the Tietze extension (applied to the one-point compactifications of $S$ and $T$ ) we may extend the $\sigma_{i}$ 's and $\tau_{i}$ 's to sections on $S$ and $T$ which vanish at infinity. Because of uppersemicontinuity of the norm it follows, then, that the inequality

$$
\left\|\omega-\sum \sigma_{i} \odot \tau_{i}\right\|<\varepsilon
$$

will hold throughout some neighborhood of $S^{\prime} \times T^{\prime}$. By a compactness argument, we may assume that the neighborhood is of the form $S^{\prime \prime} \times T^{\prime \prime}$ where $S^{\prime \prime}$ and $T^{\prime \prime}$ are compact. (Let $V$ be the original neighborhood of $S \times T$. First fix $s \in S^{\prime}$. Each point of the compact cross section $C_{s}=\{s\} \times T^{\prime}$ has a neighborhood $\widetilde{S} \times \widetilde{T} \subset V, \widetilde{S}$ and $\widetilde{T}$ compact. It follows that $C_{s}$ can be covered by the interiors of a finite number of such neighborhoods, say $S_{1} \times T_{1}, S_{2} \times T_{2}, \ldots, S_{n} \times$ $T_{n}$. If we set

$$
S_{s}=\bigcap_{k=1}^{n} S_{k} \quad \text { and } \quad T_{s}=\bigcup_{k=1}^{n} T_{k},
$$

then $S_{s} \times T_{s}$ is a neighborhood of $C_{s}$ which is inside $V$. We now let $s$ vary over $S^{\prime}$. The interiors of the sets $S_{s} \times T_{s}$ provide an open cover of $S^{\prime} \times T^{\prime}$. Hence there are a finite number of these sets, say $S_{s_{1}} \times T_{s_{1}}, S_{s_{2}} \times T_{s_{2}}, \cdots, S_{s_{m}} \times T_{s_{m}}$, whose interiors cover $S^{\prime} \times T^{\prime}$. We now set

$$
S^{\prime \prime}=\bigcup_{k=1}^{m} S_{s_{k}} \quad \text { and } \quad T^{\prime \prime}=\bigcap_{k=1}^{m} T_{s_{k}} .
$$

Then $S^{\prime \prime}$ and $T^{\prime \prime}$ are compact and $S^{\prime} \times T^{\prime} \subset$ interior of $S^{\prime \prime} \times T^{\prime \prime} \subset V$.) Since $\omega$ is zero outside $S^{\prime} \times T^{\prime}$ it follows that

$$
\left\|\sum \sigma_{i} \odot \tau_{i}\right\|<\varepsilon
$$

on $\left(S^{\prime \prime} \times T^{\prime \prime}\right) \backslash\left(S^{\prime} \times T^{\prime}\right)$. Now choose continuous functions $f: S \rightarrow[0,1]$ and $g: T \rightarrow[0,1]$ such that $f=1$ on $S^{\prime}, f=0$ off $S^{\prime \prime}, g=1$ on $T^{\prime}$, and $g=0$ off $T^{\prime \prime}$. Then it follows easily that

$$
\left\|\omega-(f \odot g) \sum \sigma_{i} \odot \tau_{i}\right\|=\left\|\omega-\sum\left(f \sigma_{i}\right) \odot\left(g \tau_{i}\right)\right\|<\varepsilon
$$


at all points of $S \times T$.

We conclude this section by constructing a tensor product of bundle maps.

THEOREM 3.6. Suppose we have four Banach bundles, $\pi_{i}: E_{\imath} \rightarrow S_{i}$, and $\rho_{i}: F_{i} \rightarrow S_{i}, i=1,2$, where $S_{1}$ and $S_{2}$ are locally compact Hausdorff. Suppose also that we are given two bundle maps $\phi_{i}: \pi_{i} \rightarrow \rho_{i}$, $i=1,2$, as indicated in the diagram:

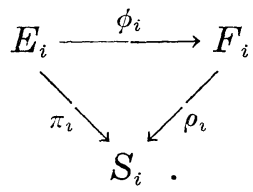

Then there exists a unique bundle map $\dot{\phi}_{1} \hat{\otimes} \dot{\phi}_{2}: \pi_{1} \hat{\otimes} \pi_{2} \rightarrow \rho_{1} \hat{\otimes} \rho_{2}$ as indicated below

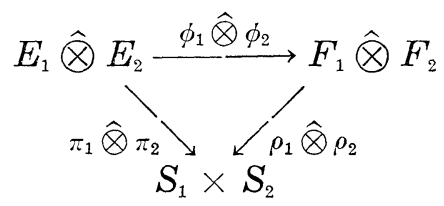

such that $\left(\dot{\phi}_{1} \hat{\otimes} \phi_{2}\right)_{\left(s_{1}, s_{2}\right)}=\left(\dot{\phi}_{1}\right)_{s_{1}} \otimes\left(\dot{\phi}_{2}\right)_{s_{2}}$, for all $\left(s_{1}, s_{2}\right) \in S_{1} \times S_{2}$. (Here, we are denoting by $\phi_{s}$ the restriction of the bundle map $\phi$ to the fiber $E_{s}$ above $s \in S$.)

Moreover, for all sections $\sigma_{i} \in \Gamma_{0}\left(\pi_{i}\right), i=1,2$, we have $\left(\phi_{1} \hat{\otimes} \phi_{2}\right) \circ$ $\left(\sigma_{1} \odot \sigma_{2}\right)=\left(\dot{\phi}_{1} \circ \sigma_{1}\right) \circledast\left(\dot{\phi}_{2} \circ \sigma_{2}\right) \in \Gamma_{0}\left(\rho_{1} \hat{\otimes} \rho_{2}\right)$, and $\left\|\dot{\phi}_{1} \hat{\otimes} \phi_{2}\right\|=\left\|\phi_{1}\right\|\left\|\phi_{2}\right\|$.

Proof. A bundle map is determined locally by its action on individual fibers, and locally the base spaces are compact. We may thus assume without loss of generality that $S_{1}$ and $S_{2}$ are, in fact, compact.

Rather than define our bundle map directly, we begin by defining the map which it induces on sections. Given a section $\omega \in \Gamma\left(\pi_{1} \hat{\otimes} \pi_{2}\right)$, define a selection (not necessarily continuous, yet) $\tilde{\omega}: S_{1} \times S_{2} \rightarrow F_{1} \hat{\otimes}$ $F_{2}$ by setting

$$
\tilde{\omega}\left(s_{1}, s_{2}\right)=\left[\left(\dot{\phi}_{1}\right)_{s_{1}} \otimes\left(\dot{\phi}_{2}\right)_{s_{2}}\right]\left(\omega\left(s_{1}, s_{2}\right)\right),
$$

for all $\left(s_{1}, s_{2}\right) \in S_{1} \times S_{2}$. It is clear that this selection $\tilde{\omega}$ is bounded by $\left\|\dot{\phi}_{1}\right\|\left\|\phi_{2}\right\|\|\omega\|$, and that the map $\omega \rightarrow \widetilde{w}$ is linear. Thus, we obtain a bounded linear map $\sim$ from $\Gamma\left(\pi_{1} \hat{\otimes} \pi_{2}\right)$ to the Banach space of all bounded selections of the bundle $\rho_{1} \hat{\otimes} \rho_{2}: F_{1} \hat{\otimes} F_{2} \rightarrow S_{1} \times S_{2}$. In fact, $\sim \operatorname{maps} \Gamma\left(\pi_{1} \hat{\otimes} \pi_{2}\right)$ into $\Gamma\left(\rho_{1} \hat{\otimes} \rho_{2}\right)$, for, if $\omega=\sigma_{1} \hat{\otimes} \sigma_{2}$, where $\sigma_{i} \epsilon$ 
$\Gamma\left(\pi_{i}\right), i=1,2$, then

$$
\begin{aligned}
\tilde{\omega}\left(s_{1}, s_{2}\right) & =\left[\left(\dot{\phi}_{1}\right)_{s_{1}} \otimes\left(\dot{\phi}_{2}\right)_{s_{2}}\right]\left(\sigma_{1}\left(s_{1}\right) \otimes \sigma_{2}\left(s_{2}\right)\right) \\
& =\phi_{1}\left(\sigma_{1}\left(s_{1}\right)\right) \otimes \phi_{2}\left(\sigma_{2}\left(s_{2}\right)\right) \\
& =\left(\left(\dot{\phi}_{1} \circ \sigma_{1}\right) \odot\left(\dot{\phi}_{2} \circ \sigma_{2}\right)\right)\left(s_{1}, s_{2}\right) .
\end{aligned}
$$

Thus, $\tilde{\omega}=\left(\dot{\phi}_{1} \circ \sigma_{1}\right) \odot\left(\dot{\phi}_{2} \circ \sigma_{2}\right) \in \Gamma\left(\rho_{1} \hat{\otimes} \rho_{2}\right)$. In the general case, $\omega$ is a uniform limit of sums of such tensor products, so that $\tilde{\omega}$ is likewise a uniform limit of sums of tensor products, and thus is in $\Gamma\left(\rho_{1} \hat{\otimes} \rho_{2}\right)$.

It is then easy to check that the map : $\Gamma\left(\pi_{1} \hat{\otimes} \pi_{2}\right) \rightarrow \Gamma\left(\rho_{1} \hat{\otimes} \rho_{2}\right)$ is a sectional representation of Gelfand type of the module $\left(\Gamma\left(\pi_{1} \hat{\otimes} \pi_{2}\right)\right.$, $C\left(S_{1}\right) \hat{\otimes} C\left(S_{2}\right)$ ); the universal mapping property of the Gelfand representation [see 5] then asserts the existence and uniqueness of a bundle map $\dot{\phi}_{1} \hat{\otimes} \dot{\phi}_{2}: \pi_{1} \hat{\otimes} \pi_{2} \rightarrow \rho_{1} \hat{\otimes} \rho_{2}$ such that

$$
\tilde{\omega}=\left(\phi_{1} \hat{\otimes} \phi_{2}\right) \circ \hat{\omega}=\left(\phi_{1} \hat{\otimes} \phi_{2}\right) \circ \omega
$$

for every $\omega \in \Gamma\left(\pi_{1} \hat{\otimes} \pi_{2}\right)$. The rest is now easy.

4. Inductive tensor products. Up to this point we have considered only projective tensor products (of both modules and bundles). In this final section we turn our attention to other cross norms, especially the inductive one. One particularly interesting fact about inductive tensor products is that there is a natural isomorphism $C_{0}(S) \hat{\hat{\otimes}} C_{0}(T) \cong C_{0}(S \times T)$, where $S$ and $T$ are locally compact Hausdorff spaces. In the present section we prove a substantial generalization of this result. If $\pi: E \rightarrow S$ and $\rho: F \rightarrow T$ are any two bundles of Banach spaces, where $S$ and $T$ are again locally compact Hausdorff, then we show that there is a bundle $\pi \hat{\hat{\otimes}} \rho: E \hat{\hat{\otimes}} F \rightarrow S \times T$, which we shall call the inductive tensor product of the given bundles, such that

(1) the stalk above any point $(s, t)$ in $S \times T$ is $E_{s} \hat{\hat{\otimes}} F_{t}$;

(2) the spaces $\Gamma_{0}(\pi) \hat{\hat{\otimes}} \Gamma_{0}(\rho)$ and $\Gamma_{0}(\pi \hat{\hat{\otimes}} \rho)$ are isometrically isomorphic. More precisely, there is a unique linear map $\phi: \Gamma_{0}(\pi) \hat{\hat{\otimes}}$ $\Gamma_{0}(\rho) \rightarrow \Gamma_{0}(\pi \hat{\hat{\otimes}} \rho)$ such that $\phi(\sigma \otimes \tau)=\sigma \circledast \tau$ for all $\sigma \in \Gamma_{0}(\pi)$ and $\tau \in \Gamma_{0}(\rho)$; this map $\phi$ is an isometric isomorphism.

The map $\dot{\rho}$ is (modulo bundle isomorphisms) the Gelfand representation of $\Gamma_{0}(\pi) \hat{\hat{\otimes}} \Gamma_{0}(\rho)$ as a Banach module over $C_{0}(S) \hat{\hat{\otimes}} C_{0}(T)$. In this respect the situation for inductive tensor products is nicer than it is for projective ones. In the projective case we again have a linear map $\phi: \Gamma_{0}(\pi) \hat{\otimes} \Gamma_{0}(\rho) \rightarrow \Gamma_{0}(\pi \hat{\otimes} \rho)$ such that $\phi(\sigma \otimes \tau)=\sigma \odot \tau$, and, again modulo bundle isomorphisms, $\phi$ is the Gelfand representation of $\Gamma_{0}(\pi) \hat{\otimes} \Gamma_{0}(\rho)$. In this case, however, $\phi$ is usually neither 
surjective nor norm-preserving (nor injective, for that matter). (See §2.)

The final theorem in this section concerns modules $(M, A)$ and $(N, B)$ which satisfy the $(\mathrm{KR})$ condition. It describes a representation of the module ( $M \hat{\hat{\otimes}} N, A \hat{\otimes} B$ ) by sections of the inductive tensor product of the canonical bundles of the given modules.

Proposition 4.1. Suppose that $(M, A)$ and $(N, B)$ are Banach modules, where $A$ and $B$ are commutative. If $\alpha$ is any uniform cross norm on $M \otimes N$, then there is a unique way in which $M \otimes_{\alpha} N$ can be made into a Banach module over $A \hat{\otimes} B$ so that

$$
(a \otimes b)(x \otimes y)=(a x) \otimes(b y)
$$

holds for all $a \in A, b \in B, x \in M$, and $y \in N$.

Proof. By the universal property of tensor products one can show that there is a unique bilinear map $P:(A \otimes B) \times(M \otimes N) \rightarrow$ $M \otimes N$ such that $P(a \otimes b, x \otimes y)=(a x) \otimes(b y)$ for all $a \in A, b \in B$, $x \in M$, and $y \in N$. We then write $P(c, z)=c z$.

Given $a \in A$ and $b \in B$, we define a linear map $\dot{\phi}_{a, b}: M \otimes N \rightarrow$ $M \otimes N$ by $\dot{\phi}_{a, b}(z)=(a \otimes b) z$ for all $z$ in $M \otimes N$. If we write $z=$ $\sum x_{i} \otimes y_{i}$, then

$$
\begin{aligned}
\alpha\left(\dot{\phi}_{a, b}(z)\right) & =\alpha\left((a \otimes b)\left(\sum x_{i} \otimes y_{i}\right)\right) \\
& =\alpha\left(\sum\left(a x_{i}\right) \otimes\left(b y_{i}\right)\right) \leqq\|a\|\|b\| \alpha\left(\sum x_{i} \otimes y_{i}\right) \\
& =\|a\|\|b\| \alpha(z) .
\end{aligned}
$$

(Since $\alpha$ is a uniform cross norm, we have more generally, $\alpha\left(\sum\left(S x_{i}\right) \otimes\right.$ $\left.\left(T y_{i}\right)\right) \leqq\|S\|\|T\| \alpha\left(\sum x_{i} \otimes y_{i}\right)$ for all bounded linear maps $S: M \rightarrow M$ and $T: N \rightarrow N$.) It follows that $\phi_{a, b}$ can be uniquely extended to a bounded linear map $\phi_{a, b}: M \bigotimes_{\alpha} N \rightarrow M \bigotimes_{\alpha} N$ with $\left\|\phi_{a, b}\right\| \leqq\|a\|\|b\|$. The map $\phi: A \times B \rightarrow B\left(M \otimes_{\alpha} N, M \bigotimes_{\alpha} N\right)$ which sends $(a, b)$ into $\phi_{a, b}$ is a bounded bilinear map of norm one or less. Hence, there is a unique linear map

$$
\tilde{\phi}: A \hat{\otimes} B \longrightarrow B\left(M \otimes_{\alpha} N, M \bigotimes_{\alpha} N\right)
$$

such that $\tilde{\phi}(a \otimes b)=\phi_{a, b}$ for all $a \in A$ and $b \in B$. Moreover, $\|\tilde{\phi}\|=$ $\|\boldsymbol{\phi}\| \leqq 1$. We now define a map

$$
P^{\prime}:(A \hat{\otimes} B) \times\left(M \otimes_{\alpha} N\right) \longrightarrow M \otimes_{\alpha} N
$$

by setting $P^{\prime}(c, z)=[\tilde{\phi}(c)](z)$. Then $P^{\prime}$ is visibly bilinear,

$$
\alpha\left(P^{\prime}(c, z)\right) \leqq\|\tilde{\phi}(c)\| \alpha(z) \leqq\|c\|^{\wedge} \alpha(z),
$$


and $P^{\prime}(a \otimes b, x \otimes y)=\phi_{a, b}(x \otimes y)=(a \otimes b)(x \otimes y)=(a x) \otimes(b y)$, i.e., $P^{\prime}$ is an extension of $P$. We then agree to write $P^{\prime}(c, z)=c z$, etc.

In the proof of the next theorem we will need a few facts about the dual space of $\Gamma_{0}(\pi)$, where $\pi: E \rightarrow S$ is a bundle of Banach spaces and $S$ is locally compact. For each point $s \in S$, there is a natural isometric embedding $\sim:\left(E_{s}\right)^{*} \rightarrow \Gamma_{0}(\pi)^{*}$ which is characterized by the identity $\widetilde{F}(\sigma)=F(\sigma(s))$ for all $F \in\left(E_{s}\right)^{*}$ and $\sigma \in \Gamma_{0}(\pi)$. If we let

$$
K=\left\{\widetilde{F}: F \in\left(E_{s}\right)^{*} \text { for some } s \in S,\|F\| \leqq 1\right\}
$$

then $K$ is a weak-* compact subset of $\Gamma_{0}(\pi)^{*}$. Moreover, if we have a convergent net of elements in $S$, say $\lim s_{\alpha}=s$, and if, for each $\alpha, F_{\alpha}$ is an element of the unit ball of $\left(E_{s_{\alpha}}\right)^{*}$, then there is a subnet of $\left\{\widetilde{F}_{\alpha}\right\}$ which converges weakly-* to a functional of the form $\widetilde{F}$, where $F$ belongs to the unit ball of $\left(E_{s}\right)^{*}$. (For more details the reader can consult the Appendix in [5].)

THEOREM 4.2. Let $\pi: E \rightarrow S$ and $\rho: F \rightarrow T$ be bundles of Banach spaces, where $S$ and $T$ are locally compact Hausdorff. Then there is a unique bundle of Banach spaces $\pi \hat{\hat{\otimes}} \rho: E \hat{\hat{\otimes}} F \rightarrow S \times T$ such that

(1) for each point $(s, t)$ in $S \times T$ the fiber above $(s, t)$ is $E_{s} \hat{\hat{\otimes}} F_{t}$;

(2) the tensor map $\otimes: E \times F \rightarrow E \hat{\hat{\otimes}} F$, which assigns to any pair $(x, y) \in E \times F$ its tensor product in $E_{\pi(x)} \hat{\hat{\otimes}} E_{\rho(y)}$, is continuous;

(3) if $\sigma: U \rightarrow E$ and $\tau: V \rightarrow F$ are local sections of $\pi$ and $\rho$ respectively, then $\sigma \odot \tau: U \times V \rightarrow E \hat{\hat{\otimes}} F$ is a local section of $\pi \hat{\hat{\otimes}} \rho$;

(4) there is a unique linear map $\phi: \Gamma_{0}(\pi) \hat{\hat{\otimes}} \Gamma_{0}(\rho) \rightarrow \Gamma_{0}(\pi \hat{\hat{\otimes}} \rho)$ such that $\phi(\sigma \otimes)=\sigma \odot \tau$ for all $\sigma \in \Gamma_{0}(\pi)$ and $\tau \in \Gamma_{0}(\rho)$; the map is an isometric isomorphism.

Proof. We let $E \hat{\hat{\otimes}} F$ be the disjoint union of the family of Banach spaces $\left\{E_{s} \hat{\hat{\otimes}} F_{t}: s \in S, t \in T\right\}$ and we let $\pi \hat{\hat{\otimes}} \rho: E \hat{\hat{\otimes}} F \rightarrow S \times T$ be the natural surjection. We denote by $\Sigma$ the set of all selections $\sigma: S \times T \rightarrow E \hat{\hat{\otimes}} F$, i.e., for all $s \in S$ and $t \in T, \sigma(s, t) \in E_{s} \hat{\otimes} F_{t}$. We also denote by $\sum_{b}$ the set of bounded selections in $\sum ; \sum_{b}$ is a Banach space with respect to the usual pointwise operations and sup norm: $\|\sigma\|=\sup \{\|\sigma(s, t)\| \hat{\wedge}: s \in S, t \in T\}$.

Using the universal property of tensor products one can show that there is a unique linear map $\theta: \Gamma_{0}(\pi) \otimes \Gamma_{0}(\rho) \rightarrow \Sigma$ such that $\theta(\sigma \otimes \tau)=\sigma \odot \tau$ for all $\sigma \in \Gamma_{0}(\pi)$ and $\tau \in \Gamma_{0}(\rho)$. We will show that $\theta$ maps $\Gamma_{0}(\pi) \otimes \Gamma_{0}(\rho)$ into $\Sigma_{b}$, and, more importantly, that $\theta$ is an isometry. In so doing, we use a characterization of inductive cross 
norms found in [1], p. 63. It will also be convenient to borrow a bit of notation from the same reference: we denote by $O X$ the closed unit ball of a Banach space $X$.

Consider, now, an arbitrary element in $\Gamma_{0}(\pi) \otimes \Gamma_{0}(\rho)$, say $\sum \sigma_{\imath} \otimes$ $\tau_{i}$. Then

$$
\begin{aligned}
& \left\|\sum \sigma_{i} \otimes \tau_{i}\right\| \hat{\imath}=\sup _{G \in \in\left(j \Gamma_{0}^{\left(\rho^{*}\right)}\right.}\left\|\sum G\left(\tau_{i}\right) \sigma_{i}\right\| \\
& =\sup _{s \in S} \sup _{G \in O \Gamma_{0}(j) *}\left\|\sum G\left(\tau_{i}\right) \sigma_{\imath}(s)\right\| \\
& \left.=\sup _{s \in S}\left\|\sum \sigma_{i}(s) \otimes \tau_{i}\right\| \hat{\wedge} \quad \text { (in } E_{s} \hat{\otimes} \Gamma_{0}(\rho)\right) \\
& =\sup _{s \in S} \sup _{F \in O\left(F_{i}^{\prime}\right)^{* *}}\left\|\sum F\left(\sigma_{i}(s)\right) \tau_{i}\right\| \\
& =\sup _{s \in S} \sup _{t \in T^{*}} \sup _{F \in O\left(E_{s}\right)^{*}}\left\|\sum F\left(\sigma_{\imath}(s)\right) \tau_{i}(t)\right\| \\
& =\sup _{s \in S} \sup _{t \in T}\left\|\sum \sigma_{i}(s) \otimes \tau_{i}(t)\right\| \hat{\wedge} \\
& =\sup _{s \in S} \sup _{t \in T^{\prime}}\left\|\sum \sigma_{\imath} \odot \tau_{i}(s, t)\right\| \hat{\wedge} \\
& =\| \sum \sigma_{i}\left(\tau_{i}\|=\| \theta\left(\sum \sigma_{i} \otimes \tau_{i}\right) \|\right. \text {. }
\end{aligned}
$$

For the same element $\sum \sigma_{\imath} \otimes \tau_{i}$ in $\Gamma_{0}(\pi) \otimes \Gamma_{0}(\rho)$ we now define a real-valued function $f$ on $S \times T$ by setting

$$
\begin{aligned}
f(s, t) & =\left\|\sum\left(\sigma_{\imath} @ \tau_{i}\right)(s, t)\right\| \hat{\wedge} \\
& =\sup _{F \in O\left(E_{s}\right)^{*}, G \in O\left(F_{s}\right)^{*}}\left|\sum\left\langle\sigma_{\imath}(s), F\right\rangle\left\langle\tau_{i}(t), G\right\rangle\right| .
\end{aligned}
$$

We will show that $f$ is upper semicontinuous on $S \times T$. Suppose that $f$ fails to be upper semicontinuous at some point $(s, t)$ in $S \times T$. Then there exists an $\varepsilon>0$ and a net $\left\{\left(s_{\alpha}, t_{\alpha}\right)\right\}$ which converges to $(s, t)$ such that $f\left(s_{\alpha}, t_{\alpha}\right) \geqq f(s, t)+\varepsilon$ for all $\alpha$. Choose $F_{\alpha} \in O\left(E_{s_{\alpha}}\right)^{*}$ and $G_{\alpha} \in O\left(F_{s_{\alpha}}\right) *$ such that

$$
\begin{aligned}
f\left(s_{\alpha}, t_{\alpha}\right) & =\left|\sum\left\langle\sigma_{i}\left(s_{\alpha}\right), F_{\alpha}\right\rangle\left\langle\tau_{i}\left(t_{\alpha}\right), G_{\alpha}\right\rangle\right| \\
& =\left|\sum\left\langle\sigma_{i}, \widetilde{F}_{\alpha}\right\rangle\left\langle\tau_{i}, \widetilde{G}_{\alpha}\right\rangle\right| .
\end{aligned}
$$

Since the functionals $\widetilde{F}_{\alpha}$ belong to the weak-* compact subset $K$ of $\Gamma_{0}(\pi)^{*}$, we may assume by passing to subnets if necessary, that $\left\{\widetilde{F}_{\alpha}\right\}$ converges weakly-* to some functional. Furthermore, the limit functional will be of the form $\widetilde{F}$ for some $F \in O\left(E_{s}\right)^{*}$. Similarly, we may assume that $\lim \widetilde{G}_{\alpha}=\widetilde{G}$ for some $G \in O\left(F_{t}\right)^{*}$. Then

$$
\begin{aligned}
f(s, t)+\varepsilon & \leqq \lim f\left(s_{\alpha}, t_{\alpha}\right) \\
& =\left|\sum\left\langle\sigma_{i}, \widetilde{F}\right\rangle\left\langle\tau_{i}, \widetilde{G}\right\rangle\right| \\
& =\left|\sum\left\langle\sigma_{i}(s), F\right\rangle\left\langle\tau_{i}(t), G\right\rangle\right| \leqq f(s, t),
\end{aligned}
$$

which is impossible. 
We can now apply an existence theorem on bundles due to Hofmann, specifically Proposition 3.6 in [4]. It follows that there is a topology on $E \hat{\hat{\otimes}} F$ which makes $\pi \hat{\hat{\otimes}} \rho: E \hat{\hat{\otimes}} F \rightarrow S \times T$ a bundle of Banach spaces and makes every selection of the form $\sum \sigma_{i} \odot \tau_{i}$ a section of the bundle. Moreover, it is easily argued that each of these sections vanishes at $\infty$. Thus, we have a isometric linear map

$$
\theta: \Gamma_{0}(\pi) \otimes \Gamma_{0}(\rho) \longrightarrow \Gamma_{0}(\pi \hat{\otimes} \rho) \text {. }
$$

By the same argument used to prove Corollary 3.5, one can show that the image of $\theta$ is dense in $\Gamma_{0}(\pi \hat{\hat{\otimes}} \rho)$. It follows then that $\theta$ can be uniquely extended to an isometric isomorphism

$$
\theta: \Gamma_{0}(\pi) \hat{\hat{\otimes}} \Gamma_{0}(\rho) \longrightarrow \Gamma_{0}(\pi \hat{\hat{\otimes}} \rho) \text {. }
$$

Properties (2) and (3) follow by previous arguments. The existence theorem of Hofmann, which we invoked in the preceding paragraph, does not guarantee the uniqueness of the topology on the fiber space. But now we can see that this topology is unique, since the topology is determined by the space of sections, which consists, in the case, of all uniform limits of selections of the form $\sum \sigma_{i} \odot \tau_{i}$.

We note three corollaries of the theorem. The first two are known. (See [1], p. 64.)

CoRollary 4.3. Let $S$ and $T$ be locally compact Hausdorff spaces. Then

$$
C_{0}(S) \hat{\hat{\otimes}} C_{0}(T) \cong C_{0}(S \times T) .
$$

More precisely, there is a unique isometric isomorphism $\theta: C_{0}(S) \hat{\hat{\otimes}}$ $C_{0}(T) \rightarrow C_{0}(S \times T)$ such that $\theta(f \otimes g)=f \odot g$, where $(f \odot g)(s, t)=$ $f(s) g(t)$.

Proof. Apply the theorem to the constant $C$-bundles over $S$ and $T$.

CoRollary 4.4. Let $S$ be a locally compact Hausdorff space and let $X$ be any Banach space. Then

$$
C_{0}(S) \hat{\hat{\otimes}} X
$$

is isometrically isomorphic to $C_{0}(S, X)$, the space of continuous $X$ valued functions on $S$ which vanish at infinity.

Proof. Let one bundle be the constant $C$-bundle over $S$ and let the second bundle have $X$ as its one and only fiber. 
CoRollary 4.5. Let $S$ and $T$ be locally compact Hausdorff spaces and let $X$ and $Y$ be Banach spaces. Then

$$
C_{0}(S, X) \hat{\hat{\otimes}} C_{0}(T, Y) \cong C_{0}(S \times T, X \hat{\hat{\otimes}} Y) .
$$

Proof. Use the bundles whose fiber spaces are $S \times X$ and $T \times Y$ with the product topologies.

Theorem 4.6. Let $(M, A)$ and $(N, B)$ be Banach modules which satisfy the (KR) condition and let $\pi: E \rightarrow S$ and $\rho: F \rightarrow T$ be their respective canonical bundles. Then the module $(M \hat{\hat{\otimes}} N, A \hat{\otimes} B)$ also satisfies the (KR) condition and there exists a unique linear map $\theta: M \hat{\hat{\otimes}} N \rightarrow \Gamma_{0}(\pi \hat{\hat{\otimes}} \rho)$ such that $\theta(x \otimes y)=\hat{x} \odot \hat{y}$ for all $x \in M$ and $y \in N$. Moreover, $\theta$ is a norm-decreasing sectional representation of Gelfand type.

Proof. The map $\theta$ is simply the inductive tensor product of the Gelfand representations ${ }^{\wedge}: M \rightarrow \Gamma_{0}(\pi)$ and ${ }^{\wedge}: N \rightarrow \Gamma_{0}(\rho)$, followed by the natural isomorphism from $\Gamma_{0}(\pi) \hat{\hat{\otimes}} \Gamma_{0}(\rho)$ into $\Gamma_{0}(\pi \hat{\hat{\otimes}} \rho)$. It is a straightforward matter to check that $\theta$ is of Gelfand type, i.e., that $\theta(c z)=\hat{c} \theta(z)$ for all $c \in A \hat{\otimes} B$ and $z \in M \hat{\hat{\otimes}} N$. (Because of continuity and bilinearity it suffices to consider monomials $c=a \otimes b$ and $z=$ $x \otimes y$, etc.) Finally, the proof that $(M \hat{\hat{\otimes}} N, A \hat{\otimes} B)$ satisfies the $(\mathrm{KR})$ condition is the same as in the projective case.

\section{REFERENCES}

1. J. Cigler, V. Losert, P. Michor, Banach Modules and Functors on Categories of Banach Spaces, Lecture Notes in Pure And Applied Mathematics, Vol. 46 Marcel Dekker, Inc., New York, 1979.

2. J. M. G. Fell, Induced representations and Banach*-algebras, Lecture Notes in Mathematics, Vol. 582, Springer-Verlag, 1977.

3. B. R. Gelbaum, Tensor products and related questions, Trans. Amer. Math. Soc., 103 (1962), 525-548.

4. K. H. Hofmann, Bundles and sheaves are equivalent in the category of Banach spaces, (to appear).

5. J. W. Kitchen, Jr. and D. A. Robbins, Gelfand representation of Banach modules, Dissertationes Mathematicae, (to appear).

6. H. Milne, Banach space properties of uniform algebras, Bull. London Math. Soc., 4 (1972), 323-326.

7. M. A. Rieffel, Induced Banach representations of Banach algebras and locally compact groups, J. Functional Analysis, 1 (1967), 443-491.

Received October 24, 1979 and in revised form July 30, 1980.

Duke University, Durham, NC 27706

AND

Trinity College,

HARTFORD, CT 06106 



\section{PACIFIC JOURNAL OF MATHEMATICS}

\section{EDITORS}

DONALD BABBITT (Managing Editor)

University of Galifornia

Los Angeles, California 90024

Hugo RossI

University of Utah

Salt Lake City, UT 84112

C. C. MOORE AND ANDREW OGG

University of California

Berkeley, CA 94720

\section{J. DugundjI}

Department of Mathematics University of Southern California

Los Angeles, California 90007

R. Finn AND J. Milgram

Stanford University

Stanford, California 94305

\section{ASSOCIATE EDITORS}

R. ARENS

E. F. BECKENBACH

B. H. NeUmanN

F. WOLF

K. YosHIDA

\section{SUPPORTING INSTITUTIONS}

UNIVERSITY OF ARIZONA

UNIVERSITY OF BRITISH COLUMBIA

CALIFORNIA INSTITUTE OF TECHNOLOGY

UNIVERSITY OF CALIFORNIA

MONTANA STATE UNIVERSITY

UNIVERSITY OF NEVADA, RENO

NEW MEXICO STATE UNIVERSITY

OREGON STATE UNIVERSITY
UNIVERSITY OF OREGON

UNIVERSITY OF SOUTHERN CALIFONIA

STANFORD UNIVERSITY

UNIVERSITY OF HAWAII

UNIVERSITY OF TOKYO

UNIVERSITY OF UTAH

WASHINGTON STATE UNIVERSITY

UNIVERSITY OF WASHINGTON 


\section{Pacific Journal of Mathematics}

\section{Vol. 94, No. 1 \\ May, 1981}

Willy Brandal, Conditions for being an FGC domain $\ldots \ldots \ldots \ldots \ldots \ldots \ldots$

Allan Calder and Frank Williams, Incompressibility of maps and the

homotopy invariance of Čech cohomology $\ldots \ldots \ldots \ldots \ldots \ldots \ldots \ldots \ldots$

Jacques Chaumat, Quelques propriétés du couple d'espaces vectoriels

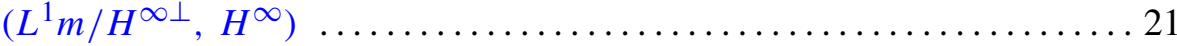

Manfred Droste and Rüdiger Göbel, Products of conjugate permutations . . 47

Jean Esterle, Rates of decrease of sequences of powers in commutative

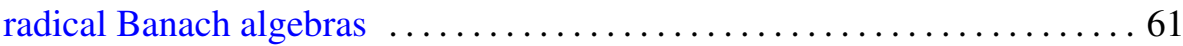

Allan Fryant, Ultraspherical expansions and pseudo analytic functions . . . 83

John Hannah, Homogenization of regular rings of bounded index. II . . . . . 107

Shigeru Haruki, On the theorem of S. Kakutani-M. Nagumo and J. L. Walsh

for the mean value property of harmonic and complex polynomials $\ldots .113$

Hugh M. Hilden, Representations of homology 3-spheres $\ldots \ldots \ldots \ldots \ldots 125$

Craig Huneke, A characterization of locally Macaulay completions . . . . . 131

Takesi Isiwata, Closed ultrafilters and realcompactness ................. 139

Joseph Weston Kitchen, Jr. and David A. Robbins, Tensor products of

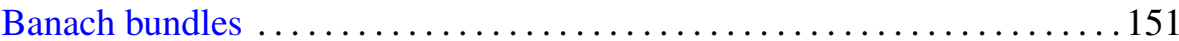

Allan J. Kroopnick, Note on bounded $L^{p}$-solutions of a generalized Liénard

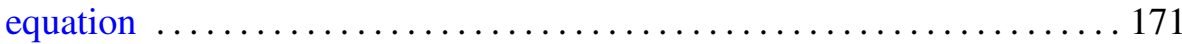

Ajay Kumar and Ajit Kaur Chilana, Spectral synthesis in products and quotients of hypergroups

Charles Livingston, Homology cobordisms of 3-manifolds, knot

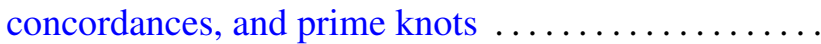

Hans Opolka, Projective representations of finite groups in cyclotomic fields

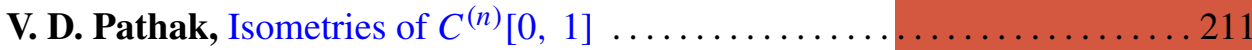

Mark Allan Pinsky, On the spectrum of Cartan-Hadamard manifolds . . . . . 2223

Judith Roitman, The number of automorphisms of an atomic Boolean

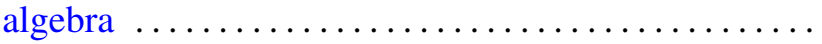

Kai Wang, Locally smooth torus group actions on integral cohomology complex projective spaces 